\title{
A WEAK TYPE ESTIMATE FOR ROUGH SINGULAR INTEGRALS
}

\author{
ANDREI K. LERNER
}

\begin{abstract}
We obtain a weak type $(1,1)$ estimate for a maximal operator associated with the classical rough homogeneous singular integrals $T_{\Omega}$. In particular, this provides a different approach to a sparse domination for $T_{\Omega}$ obtained recently by Conde-Alonso, Culiuc, Di Plinio and Ou [ [5].
\end{abstract}

\section{INTRODUCTION}

In this paper we consider a class of rough homogeneous singular integrals defined by

$$
T_{\Omega} f(x)=\text { p.v. } \int_{\mathbb{R}^{n}} f(x-y) \frac{\Omega(y /|y|)}{|y|^{n}} d y,
$$

with $\Omega \in L^{q}\left(S^{n-1}\right), q \geq 1$, having zero average.

Calderón and Zygmund [2] proved that if $\Omega \in L \log L\left(S^{n-1}\right)$, then $T_{\Omega}$ is bounded on $L^{p}$ for all $1<p<\infty$. The weak type $(1,1)$ of $T_{\Omega}$ was established by Christ [3] and Hofmann [10] in the case $n=2$ and $\Omega \in L^{q}\left(S^{1}\right), q>1$, and by Christ and Rubio de Francia [4 for $\Omega \in L \log L\left(S^{1}\right)$. Finally Seeger [17] proved that $T_{\Omega}$ is weak $(1,1)$ bounded for $\Omega \in L \log L\left(S^{n-1}\right)$ in all dimensions.

Notice that contrary to singular integrals with smooth kernels, for rough singular integrals the question whether the maximal singular integral operator

$$
T_{\Omega}^{\star} f(x)=\sup _{\varepsilon>0}\left|T_{\Omega}\left(f \chi_{\{|\cdot|>\varepsilon\}}\right)(x)\right|
$$

is of weak type $(1,1)$ is still open even in the case when $\Omega \in L^{\infty}\left(S^{n-1}\right)$.

On the other hand, weak type estimates of maximal truncations are important for the so-called sparse domination. Sparse bounds for different operators is a recent trend in Harmonic Analysis (see, e.g., [1, 5, 6, 7, 12, 13, 14, 15], and this list is far from complete). By sparse

2010 Mathematics Subject Classification. 42B20, 42B25.

Key words and phrases. Rough singular integrals, sparse bounds, maximal operators.

The author was supported by the Israel Science Foundation (grant No. 447/16). 
bounds one typically means a domination of the bilinear form $|\langle T f, g\rangle|$ (for a given operator $T$ ) by

$$
\Lambda(f, g)=\sum_{Q \in \mathcal{S}}\langle f\rangle_{r, Q}\langle g\rangle_{s, Q}|Q|
$$

with suitable $1 \leq r, s<\infty$, where $\langle f\rangle_{p, Q}=\left(\frac{1}{|Q|} \int_{Q}|f|^{p}\right)^{1 / p}$, and $\mathcal{S}$ is a sparse family of cubes from $\mathbb{R}^{n}$. We say that $\mathcal{S}$ is $\eta$-sparse, $0<\eta \leq 1$, if for every cube $Q \in \mathcal{S}$, there exists a measurable set $E_{Q} \subset Q$ such that $\left|E_{Q}\right| \geq \eta|Q|$, and the sets $\left\{E_{Q}\right\}_{Q \in \mathcal{S}}$ are pairwise disjoint. The advantage of sparse bounds is that they easily imply quantitative weighted estimates in terms of the Muckenhoupt and reverse Hölder constants.

In [15], the following principle was established: if $T$ is a sublinear operator of weak type $(p, p)$, and the maximal operator

$$
M_{T} f(x)=\sup _{Q \ni x}\left\|T\left(f \chi_{\mathbb{R}^{n} \backslash 3 Q}\right)\right\|_{L^{\infty}(Q)}
$$

is of weak type $(r, r)$, for some $1 \leq p \leq r<\infty$, then $T$ is dominated pointwise by the sparse operator $\sum_{Q \in \mathcal{S}}\langle f\rangle_{r, Q} \chi_{Q}$.

While this principle perfectly works for smooth singular integrals, it seems to be not as useful for rough singular integrals $T_{\Omega}$. Indeed, in this case the lack of smoothness does not allow to handle the $L^{\infty}$ norm appearing in the definition of $M_{T_{\Omega}}$ in an efficient way. Observe also that for $\Omega \in L^{\infty}\left(S^{n-1}\right)$ the operator $M_{T_{\Omega}}$ may be as large as $T_{\Omega}^{\star}$, and therefore the weak type $(1,1)$ for $M_{T_{\Omega}}$ is a difficult open question.

Recently, Conde-Alonso, Culiuc, Di Plinio and Ou [5] obtained another sparse domination principle, not relying on the end-point weak type estimates of maximal truncations. This principle was effectively applied to rough singular integrals. For example, if $\Omega \in L^{\infty}$, the following estimate in [5] was proved for all $1<p<\infty$ :

$$
\left|\left\langle T_{\Omega} f, g\right\rangle\right| \leq C_{n} p^{\prime}\|\Omega\|_{L^{\infty}\left(S^{n-1}\right)} \sup _{\mathcal{S}} \sum_{Q \in \mathcal{S}}\langle f\rangle_{p, Q}\langle g\rangle_{1, Q}|Q|,
$$

where $p^{\prime}=\frac{p}{p-1}$. This estimate recovers the quantitative weighted bound

$$
\left\|T_{\Omega}\right\|_{L^{2}(w) \rightarrow L^{2}(w)} \leq C_{n}\|\Omega\|_{L^{\infty}\left(S^{n-1}\right)}[w]_{A_{2}}^{2},
$$

obtained earlier by Hytönen, Roncal and Tapiola [11.

The dependencies on $[w]_{A_{2}}$ in (1.2) and on $p$ in (1.1) when $p \rightarrow 1$ are closely related. At this time, we do not know whether the quadratic dependence on $[w]_{A_{2}}$ in $(1.2)$ can be improved. By this reason, it is also unknown whether the dependence on $p$ in (1.1) is sharp.

In this paper we present a different approach to (1.1) and (1.2) based on weak type estimates of suitable maximal operators. We believe that 
this approach is of independent interest in the theory of the rough singular integrals.

Given an operator $T$, define the maximal operator $M_{\lambda, T}$ by

$$
M_{\lambda, T} f(x)=\sup _{Q \ni x}\left(T\left(f \chi_{\mathbb{R}^{n} \backslash 3 Q}\right) \chi_{Q}\right)^{*}(\lambda|Q|) \quad(0<\lambda<1),
$$

where the supremum is taken over all cubes $Q \subset \mathbb{R}^{n}$ containing the point $x$, and $f^{*}$ denotes the non-increasing rearrangement of $f$.

Assume that $T$ is of weak type $(1,1)$. Then it is easy to show (just using that $T\left(f \chi_{\mathbb{R}^{n} \backslash 3 Q}\right)=T f-T\left(f \chi_{3 Q}\right)$ along with the standard estimates of the maximal operators) that $M_{\lambda, T}$ is of weak type $(1,1)$ too, and

$$
\left\|M_{\lambda, T}\right\|_{L^{1} \rightarrow L^{1, \infty}} \leq \frac{C_{n}}{\lambda}\|T\|_{L^{1} \rightarrow L^{1, \infty}} \quad(0<\lambda<1) .
$$

On the other hand, $M_{\lambda, T} \uparrow M_{T}$ as $\lambda \rightarrow 0$. Therefore, the weak type $(1,1)$ of $M_{T}$ (which, by [15], leads to the best possible sparse domination of $T$ ) is equivalent to the weak type $(1,1)$ of $M_{\lambda, T}$ with the $\left\|M_{\lambda, T}\right\|_{L^{1} \rightarrow L^{1, \infty}}$ norm bounded in $\lambda$. These observations raise a natural question about the sharp dependence of $\left\|M_{\lambda, T}\right\|_{L^{1} \rightarrow L^{1, \infty}}$ on $\lambda$ for a given operator $T$ of weak type $(1,1)$. More generally, if $T$ is of weak type $(p, p)$, one can ask about the sharp dependence of $\left\|M_{\lambda, T}\right\|_{L^{p} \rightarrow L^{p, \infty}}$ on $\lambda$.

The main result of this paper is the following estimate for rough homogeneous singular integrals $T_{\Omega}$.

Theorem 1.1. If $\Omega \in L^{\infty}\left(S^{n-1}\right)$, then

$$
\left\|M_{\lambda, T_{\Omega}}\right\|_{L^{1} \rightarrow L^{1, \infty}} \leq C_{n}\|\Omega\|_{L^{\infty}\left(S^{n-1}\right)}\left(1+\log \frac{1}{\lambda}\right) \quad(0<\lambda<1) .
$$

The proof of Theorem 1.1 is given in the next Section. In Section 3, we obtain a sparse domination principle, where the operator $M_{\lambda, T}$ plays an important role. In particular, we will show that (1.3) implies the sparse bound (1.1).

Notice that any improvement of the logarithmic dependence in (1.3) would lead to the corresponding improvement of the dependence on $p$ when $p \rightarrow 1$ in (1.1). Therefore, by the reasons discussed above, we do not know whether the logarithmic dependence in (1.3) can be improved.

\section{Proof of Theorem 1.1}

2.1. An overview of the proof. As we have mentioned before, for smooth singular integrals one can use the trivial estimate $M_{\lambda, T} \leq M_{T}$, which yields the $L^{1} \rightarrow L^{1, \infty}$ bound with no dependence on $\lambda$. This simple idea suggests to approximate a rough singular integral $T_{\Omega}$ by 
smooth ones. Given $0<\varepsilon<1$, we decompose

$$
T_{\Omega}=T_{\Omega_{\varepsilon}}+T_{\Omega-\Omega_{\varepsilon}},
$$

where $T_{\Omega_{\varepsilon}}$ is a smooth singular integral to which the standard CalderónZygmund theory is applicable, and $\left\|T_{\Omega-\Omega_{\varepsilon}}\right\|_{L^{2} \rightarrow L^{2}}$ satisfies a good estimate in terms of $\varepsilon$ when $\varepsilon \rightarrow 0$. Then

$$
M_{\lambda, T_{\Omega}} \leq M_{T_{\Omega_{\varepsilon}}}+M_{\lambda, T_{\Omega-\Omega_{\varepsilon}}} .
$$

For the smooth part $T_{\Omega_{\varepsilon}}$ we use a very similar analysis to what was done by Hytönen, Roncal and Tapiola [11], namely, we show that the kernel of $T_{\Omega_{\varepsilon}}$ is Dini-continuous tracking the Dini constant, which implies

$$
\left\|M_{T_{\Omega_{\varepsilon}}}\right\|_{L^{1} \rightarrow L^{1, \infty}} \leq C_{n}\|\Omega\|_{L^{\infty}\left(S^{n-1}\right)} \log \frac{2}{\varepsilon} \quad(0<\varepsilon<1) .
$$

The non-smooth part $T_{\Omega-\Omega_{\varepsilon}}$ is more complicated. The only fact that $T_{\Omega-\Omega_{\varepsilon}}$ is a singular integral with small $L^{2}$ norm in terms of $\varepsilon$ is not enough in order to obtain a good estimate for $\left\|M_{\lambda, T_{\Omega-\Omega_{\varepsilon}}}\right\|_{L^{1} \rightarrow L^{1, \infty}}$ in terms of $\varepsilon$ and $\lambda$. However, we can keep $\Omega_{\varepsilon}$ in (2.1) to be homogeneous. This allows to apply to $T_{\Omega-\Omega_{\varepsilon}}$ the deep machinery developed by Seeger in [17]. Combining it with several other ingredients, we obtain

$$
\left\|M_{\lambda, T_{\Omega-\Omega_{\varepsilon}}}\right\|_{L^{1} \rightarrow L^{1, \infty}} \leq C_{n}\|\Omega\|_{L^{\infty}\left(S^{n-1}\right)}\left(\frac{\varepsilon^{1 / 2}}{\lambda}+\log \frac{2}{\varepsilon}\right) .
$$

It remains to optimize the obtained estimates with respect to $\varepsilon$, namely, we take $\varepsilon=\lambda^{2}$.

The details follow in next subsections.

2.2. Main splitting. Denote

$$
\Omega_{0}(x)=\frac{\Omega(x /|x|)}{|x|^{n}} \chi_{\{1 \leq|x| \leq 2\}}(x) .
$$

Let $\varphi \in C^{\infty}, \operatorname{supp} \varphi \subset\{|x|<1\}$ and $\int \varphi=1$. For $\varepsilon>0$, set

$$
\Omega_{\varepsilon}(\theta)=\frac{1}{\log 2} \int_{0}^{\infty} \Omega_{0} * \varphi_{\varepsilon}(t \theta) t^{n-1} d t \quad\left(\theta \in S^{n-1}\right),
$$

where $\varphi_{\varepsilon}(x)=\frac{1}{\varepsilon^{n}} \varphi(x / \varepsilon)$.

We split $T_{\Omega}$ as follows: $T_{\Omega}=T_{\Omega_{\varepsilon}}+T_{\Omega-\Omega_{\varepsilon}}$.

Lemma 2.1. For every $0<\alpha<1$,

$$
\left\|T_{\Omega-\Omega_{\varepsilon}}\right\|_{L^{2} \rightarrow L^{2}} \leq C_{\alpha, n}\|\Omega\|_{L^{\infty}\left(S^{n-1}\right)} \varepsilon^{\alpha} \quad(0<\varepsilon<1) .
$$

Proof. Observe that the kernel of $T_{\Omega-\Omega_{\varepsilon}}$ is given by

$$
\Psi_{\varepsilon}(x)=\frac{\left(\Omega-\Omega_{\varepsilon}\right)(x /|x|)}{|x|^{n}}=\frac{1}{\log 2} \int_{0}^{\infty}\left(\Omega_{0}-\Omega_{0} * \varphi_{\varepsilon}\right)(t x) t^{n-1} d t .
$$


Hence, by Plancherel's theorem, it suffices to show that

$$
\left\|\widehat{\Psi}_{\varepsilon}\right\|_{L^{\infty}} \leq C_{\alpha, n}\|\Omega\|_{L^{\infty}\left(S^{n-1}\right)} \varepsilon^{\alpha},
$$

where the Fourier transform is taken in the appropriate principal value sense.

We will use the following well known estimate (see [8]):

$$
\left|\widehat{\Omega}_{0}(\xi)\right| \leq C_{\alpha, n}\|\Omega\|_{L^{\infty}\left(S^{n-1}\right)} \min \left(|\xi|,|\xi|^{-\alpha}\right) \quad(0<\alpha<1) .
$$

Also, since $\int \varphi=1$, we have

$$
|\widehat{\varphi}(\xi)-1| \leq C \min (|\xi|, 1),
$$

with some absolute $C>0$. Combining these estimates yields

$$
\begin{aligned}
& \left|\widehat{\Psi}_{\varepsilon}(\xi)\right| \leq \frac{1}{\log 2} \int_{0}^{\infty}\left|\widehat{\Omega}_{0}(\xi / t) \| \widehat{\varphi}(\varepsilon \xi / t)-1\right| \frac{d t}{t} \\
& \leq C\|\Omega\|_{L^{\infty}} \int_{0}^{\infty} \min \left(|\xi / t|,|\xi / t|^{-\alpha}\right) \min (|\varepsilon \xi / t|, 1) \frac{d t}{t} \\
& =C\|\Omega\|_{L^{\infty}} \int_{0}^{\infty} \min \left(1 / t, t^{\alpha}\right) \min (\varepsilon / t, 1) \frac{d t}{t} \\
& \leq C\|\Omega\|_{L^{\infty}}\left(\int_{0}^{1} \min (\varepsilon / t, 1) \frac{d t}{t^{1-\alpha}}+\varepsilon\right) \leq C\|\Omega\|_{L^{\infty}} \varepsilon^{\alpha},
\end{aligned}
$$

which proves (2.2).

2.3. Calderón-Zygmund theory of $T_{\Omega_{\varepsilon}}$. Let $T f=$ p.v. $f * K$ be $L^{2}$ bounded with $K$ satisfying $|K(x)| \leq \frac{C_{K}}{|x|^{n}}$ and

$$
|K(x-y)-K(x)| \leq \omega(|y| /|x|) \frac{1}{|x|^{n}} \quad(|y|<|x| / 2),
$$

where

$$
[\omega]_{\text {Dini }}=\int_{0}^{1} \omega(t) \frac{d t}{t}<\infty .
$$

It was proved in [15, Lemma 3.2] that

$$
M_{T} f(x) \leq C_{n}\left([\omega]_{\text {Dini }}+C_{K}\right) M f(x)+T^{\star} f(x),
$$

where $M$ is the Hardy-Littlewood maximal operator, and $T^{\star}$ is the maximal singular integral. The classical proof (see, e.g., [9, Ch. 4.3]) shows that

$$
\left\|T^{\star}\right\|_{L^{1} \rightarrow L^{1, \infty}} \leq C_{n}\left(\|T\|_{L^{2} \rightarrow L^{2}}+C_{K}+[\omega]_{\text {Dini }}\right) .
$$

This, along with the previous estimate, implies

$$
\left\|M_{T}\right\|_{L^{1} \rightarrow L^{1, \infty}} \leq C_{n}\left(\|T\|_{L^{2} \rightarrow L^{2}}+C_{K}+[\omega]_{\text {Dini }}\right) .
$$


Lemma 2.2. The operator $T_{\Omega_{\varepsilon}}$ satisfies

$$
\left\|M_{T_{\Omega_{\varepsilon}}}\right\|_{L^{1} \rightarrow L^{1, \infty}} \leq C_{n}\|\Omega\|_{L^{\infty}\left(S^{n-1}\right)} \log \frac{2}{\varepsilon} \quad(0<\varepsilon<1) .
$$

Proof. Observe that for $0<\varepsilon<1, \operatorname{supp} \Omega_{0} * \varphi_{\varepsilon} \subset\{|x| \leq 3\}$. Also,

$$
\left\|\Omega_{0} * \varphi_{\varepsilon}\right\|_{L^{\infty}} \leq\left\|\Omega_{0}\right\|_{L^{\infty}}\left\|\varphi_{\varepsilon}\right\|_{L^{1}} \leq C\|\Omega\|_{L^{\infty}} .
$$

Therefore, setting $K_{\varepsilon}(x)=\frac{\Omega_{\varepsilon}(x /|x|)}{|x|^{n}}$, we obtain

$$
C_{K_{\varepsilon}}=\left\|\Omega_{\varepsilon}\right\|_{L^{\infty}} \leq \frac{1}{\log 2}\left\|\Omega_{0} * \varphi_{\varepsilon}\right\|_{L^{\infty}} \int_{0}^{3} t^{n-1} d t \leq C_{n}\|\Omega\|_{L^{\infty}} .
$$

This, along with the standard $L^{2}$ bound (see [2]), implies

$$
\left\|T_{\Omega_{\varepsilon}}\right\|_{L^{2} \rightarrow L^{2}} \leq C_{n}\left\|\Omega_{\varepsilon}\right\|_{L^{\infty}} \leq C_{n}\|\Omega\|_{L^{\infty}} .
$$

Further, using that

$$
K_{\varepsilon}(x)=\frac{1}{\log 2} \int_{0}^{\infty} \Omega_{0} * \varphi_{\varepsilon}(t x) t^{n-1} d t,
$$

we obtain

$$
\begin{aligned}
\left|\nabla K_{\varepsilon}(x)\right| & =\frac{1}{\varepsilon} \frac{1}{\log 2} \int_{0}^{\infty} \Omega_{0} *(\nabla \varphi)_{\varepsilon}(t x) t^{n} d t \\
& =\frac{1}{\varepsilon} \frac{1}{|x|^{n+1}} \frac{1}{\log 2} \int_{0}^{\infty} \Omega_{0} *(\nabla \varphi)_{\varepsilon}(t x /|x|) t^{n} d t .
\end{aligned}
$$

From this and from the same argument as used in the proof of (2.5),

$$
\left|\nabla K_{\varepsilon}(x)\right| \leq \frac{C_{n}}{\varepsilon}\|\Omega\|_{L^{\infty}} \frac{1}{|x|^{n+1}} .
$$

Therefore, by the mean value theorem,

$$
\left|K_{\varepsilon}(x-y)-K_{\varepsilon}(x)\right| \leq \frac{C_{n}}{\varepsilon}\|\Omega\|_{L^{\infty}} \frac{|y|}{|x|} \frac{1}{|x|^{n}} \quad(|y|<|x| / 2) .
$$

Also, by (2.5),

$$
\left|K_{\varepsilon}(x-y)-K_{\varepsilon}(x)\right| \leq C_{n}\|\Omega\|_{L^{\infty}} \frac{1}{|x|^{n}} \quad(|y|<|x| / 2) .
$$

Hence, $K_{\varepsilon}$ satisfies (2.3) with

$$
\omega(t)=C_{n}\|\Omega\|_{L^{\infty}} \min (1, t / \varepsilon),
$$

which implies

$$
[\omega]_{\text {Dini }} \leq C_{n}\|\Omega\|_{L^{\infty}} \log \frac{2}{\varepsilon} .
$$

This, along with (2.5), (2.6) and (2.4), completes the proof. 
2.4. The key estimate. In order to handle the rough part $T_{\Omega-\Omega_{\varepsilon}}$, we will prove the following lemma which can be stated for a general rough homogeneous singular integral $T_{\Omega}$ with $\Omega \in L^{\infty}\left(S^{n-1}\right)$.

Lemma 2.3. There exists $C_{n}>0$ such that for every $0<\delta \leq 1$,

$$
\left\|M_{\lambda, T_{\Omega}}\right\|_{L^{1} \rightarrow L^{1, \infty}} \leq C_{n}\left(\frac{\delta}{\lambda}+\log \frac{2}{\delta}\right) \max \left(\|\Omega\|_{L^{\infty}\left(S^{n-1}\right)}, \frac{\left\|T_{\Omega}\right\|_{L^{2} \rightarrow L^{2}}}{\delta}\right) .
$$

Before proving Lemma 2.3, let us show how to complete the proof of Theorem 1.1.

By (2.5),

$$
\left\|\Omega-\Omega_{\varepsilon}\right\|_{L^{\infty}} \leq C_{n}\|\Omega\|_{L^{\infty}} .
$$

This, combined with Lemmata 2.1 (where we take $\alpha=1 / 2$ ) and 2.3 , implies

$$
\left\|M_{\lambda, T_{\Omega-\Omega_{\varepsilon}}}\right\|_{L^{1} \rightarrow L^{1, \infty}} \leq C_{n}\|\Omega\|_{L^{\infty}}\left(\frac{\delta}{\lambda}+\log \frac{2}{\delta}\right) \max \left(1, \varepsilon^{1 / 2} / \delta\right) .
$$

Taking here $\delta=\varepsilon^{1 / 2}$, we obtain

$$
\left\|M_{\lambda, T_{\Omega-\Omega_{\varepsilon}}}\right\|_{L^{1} \rightarrow L^{1, \infty}} \leq C_{n}\|\Omega\|_{L^{\infty}}\left(\frac{\varepsilon^{1 / 2}}{\lambda}+\log \frac{2}{\varepsilon}\right) .
$$

Since

$$
M_{\lambda, T_{\Omega}} f(x) \leq M_{T_{\Omega_{\varepsilon}}}(x)+M_{\lambda, T_{\Omega-\Omega_{\varepsilon}}} f(x),
$$

by Lemma 2.2 cobmined with (2.7),

$$
\begin{aligned}
\left\|M_{\lambda, T_{\Omega}}\right\|_{L^{1} \rightarrow L^{1, \infty}} & \leq 2\left(\left\|M_{T_{\Omega_{\varepsilon}}}\right\|_{L^{1} \rightarrow L^{1, \infty}}+\left\|M_{\lambda, T_{\Omega-\Omega_{\varepsilon}}}\right\|_{L^{1} \rightarrow L^{1, \infty}}\right) \\
& \leq C_{n}\|\Omega\|_{L^{\infty}}\left(\frac{\varepsilon^{1 / 2}}{\lambda}+\log \frac{2}{\varepsilon}\right) .
\end{aligned}
$$

Finally, we take here $\varepsilon=\lambda^{2}$, and this completes the proof of Theorem 1.1 .

We turn now to the proof of Lemma 2.3.

2.5. A reduction to dyadic case. It will be convenient to work with a dyadic version of $M_{\lambda, T_{\Omega}}$. We first state several preliminary facts about dyadic lattices.

Given a cube $Q_{0} \subset \mathbb{R}^{n}$, let $\mathcal{D}\left(Q_{0}\right)$ denote the set of all dyadic cubes with respect to $Q_{0}$, that is, the cubes obtained by repeated subdivision of $Q_{0}$ and each of its descendants into $2^{n}$ congruent subcubes.

A dyadic lattice $\mathscr{D}$ in $\mathbb{R}^{n}$ is any collection of cubes such that

(i) if $Q \in \mathscr{D}$, then each child of $Q$ is in $\mathscr{D}$ as well;

(ii) every 2 cubes $Q^{\prime}, Q^{\prime \prime} \in \mathscr{D}$ have a common ancestor, i.e., there exists $Q \in \mathscr{D}$ such that $Q^{\prime}, Q^{\prime \prime} \in \mathcal{D}(Q)$;

(iii) for every compact set $K \subset \mathbb{R}^{n}$, there exists a cube $Q \in \mathscr{D}$ containing $K$. 
For this definition, as well as for the next Theorem, we refer to [16].

Theorem 2.4. (The Three Lattice Theorem) For every dyadic lattice $\mathscr{D}$, there exist $3^{n}$ dyadic lattices $\mathscr{D}^{(1)}, \ldots, \mathscr{D}^{\left(3^{n}\right)}$ such that

$$
\{3 Q: Q \in \mathscr{D}\}=\cup_{j=1}^{3^{n}} \mathscr{D}^{(j)}
$$

and for every cube $Q \in \mathscr{D}$ and $j=1, \ldots, 3^{n}$, there exists a unique cube $R \in \mathscr{D}^{(j)}$ of sidelength $\ell_{R}=3 \ell_{Q}$ containing $Q$.

Turn now to the definition of $M_{\lambda, T_{\Omega}}$. Fix a dyadic lattice $\mathscr{D}$. Let $Q$ be an arbitrary cube containing the point $x$. There exists a cube $R \in \mathscr{D}$ containing the center of $Q$ and such that $\ell_{Q} / 2<\ell_{R} \leq \ell_{Q}$ (by $\ell_{Q}$ we denote the sidelength of $Q$ ). Then $Q \subset 3 R$, and hence $3 Q \subset 9 R$. For every $\xi \in Q$,

$$
\left|T_{\Omega}\left(f \chi_{9 R \backslash 3 Q}\right)(\xi)\right| \leq C_{n}\|\Omega\|_{L^{\infty}} \frac{1}{|9 R|} \int_{9 R}|f| \leq C_{n}\|\Omega\|_{L^{\infty}} M f(x) .
$$

Hence,

$$
\begin{aligned}
& \left(T_{\Omega}\left(f \chi_{\mathbb{R}^{n} \backslash 3 Q}\right) \chi_{Q}\right)^{*}(\lambda|Q|) \\
& \leq\left(T_{\Omega}\left(f \chi_{\mathbb{R}^{n} \backslash 9 R}\right) \chi_{3 R}\right)^{*}(\lambda|R|)+C_{n}\|\Omega\|_{L^{\infty}} M f(x) .
\end{aligned}
$$

By Theorem 2.4 there exists a dyadic lattice $\mathscr{D}^{(j)}, j=1, \ldots 3^{n}$ such that $3 R \in \mathscr{D}^{(j)}$. Applying Theorem 2.4 again, we obtain that there are dyadic lattices $\mathscr{D}^{(j, i)}$ such that

$$
\left\{3 Q: Q \in \mathscr{D}^{(j)}\right\}=\cup_{i=1}^{3^{n}} \mathscr{D}^{(j, i)}
$$

Hence, setting

$$
\mathscr{E}^{(j, i)}=\left\{Q \in \mathscr{D}^{(j)}: 3 Q \in \mathscr{D}^{(j, i)}\right\},
$$

by (2.8), we obtain

$$
M_{\lambda, T_{\Omega}} f(x) \leq \sum_{i, j=1}^{3^{n}} M_{\lambda / 3^{n}, T_{\Omega}}^{\mathscr{E}(j, i)} f(x)+C_{n}\|\Omega\|_{L^{\infty}} M f(x),
$$

where

$$
M_{\lambda, T_{\Omega}}^{\mathscr{E}^{(j, i)}} f(x)=\sup _{Q \ni x: Q \in \mathscr{D}^{(j)}, 3 Q \in \mathscr{D}(j, i)}\left(T_{\Omega}\left(f \chi_{\mathbb{R}^{n} \backslash 3 Q}\right) \chi_{Q}\right)^{*}(\lambda|Q|) .
$$

Fix now two dyadic lattices $\mathscr{D}$ and $\mathscr{D}^{\prime}$. Let $\mathcal{F}$ be any finite family of cubes $Q$ from $\mathscr{D}$ such that $3 Q \in \mathscr{D}^{\prime}$. By (2.9), by the weak type $(1,1)$ of $M$, and by the monotone convergence theorem, it suffices to prove Lemma 2.3 for the dyadic version of $M_{\lambda, T_{\Omega}}$ defined by

$$
M_{\lambda, T_{\Omega}}^{\mathcal{F}} f(x)= \begin{cases}\max _{Q \ni x, Q \in \mathcal{F}}\left(T_{\Omega}\left(f \chi_{\mathbb{R}^{n} \backslash 3 Q}\right) \chi_{Q}\right)^{*}(\lambda|Q|), & x \in \cup_{Q \in \mathcal{F}} Q \\ 0, & \text { otherwise. }\end{cases}
$$


2.6. The Calderón-Zygmund splitting. Let $f \in L^{1}\left(\mathbb{R}^{n}\right)$ and let $\alpha>0$. Apply the Calderón-Zygmund decomposition to $f$ at height $A \alpha$ formed by the cubes from $\mathscr{D}^{\prime}$, where $A>0$ will be specified later. To be more precise, let $M^{\mathscr{D}^{\prime}}$ be the dyadic maximal operator with respect to $\mathscr{D}^{\prime}$. Let $\mathcal{P}$ be a family of the maximal pairwise disjoint cubes forming the set $\left\{x: M^{\mathscr{D}^{\prime}} f(x)>A \alpha\right\}$. For a cube $P \in \mathcal{P}$ set $b_{P}=\left(f-\frac{1}{|P|} \int_{P} f\right) \chi_{P}$. Next, let $b=\sum_{P \in \mathcal{P}} b_{P}$ and $g=f-b$. We have

$$
\left|\left\{M_{\lambda, T_{\Omega}}^{\mathcal{F}} f>\alpha\right\}\right| \leq\left|\left\{M_{\lambda / 2, T_{\Omega}}^{\mathcal{F}} g>\alpha / 2\right\}\right|+\left|\left\{M_{\lambda / 2, T_{\Omega}}^{\mathcal{F}} b>\alpha / 2\right\}\right|
$$

(notice that here we have used the standard property of the rearrangement saying that $\left.(f+g)^{*}(t) \leq f^{*}(t / 2)+g^{*}(t / 2)\right)$.

For the good part, we will use the following simple lemma.

Lemma 2.5. Assume that $T$ is a sublinear, $L^{2}$ bounded operator. Then

$$
\left\|M_{\lambda, T} f\right\|_{L^{2, \infty}} \leq \frac{C_{n}}{\lambda^{1 / 2}}\|T\|_{L^{2} \rightarrow L^{2}}\|f\|_{L^{2}} \quad(0<\lambda<1) .
$$

Proof. Let $x \in Q$. Then, by Chebyshev's inequality,

$$
\begin{aligned}
& \left(T\left(f \chi_{\mathbb{R}^{n} \backslash 3 Q}\right) \chi_{Q}\right)^{*}(\lambda|Q|) \\
& \leq\left((T f) \chi_{Q}\right)^{*}(\lambda|Q| / 2)+\left(T\left(f \chi_{3 Q}\right)\right)^{*}(\lambda|Q| / 2) \\
& \leq \frac{C_{n}}{\lambda^{1 / 2}}\left(M_{2}(T f)(x)+\|T\|_{L^{2} \rightarrow L^{2}} M_{2} f(x)\right),
\end{aligned}
$$

where $M_{2} f(x)=M\left(|f|^{2}\right)(x)^{1 / 2}$. Therefore,

$$
M_{\lambda, T} f(x) \leq \frac{C_{n}}{\lambda^{1 / 2}}\left(M_{2}(T f)(x)+\|T\|_{L^{2} \rightarrow L^{2}} M_{2} f(x)\right),
$$

which, along with the $L^{2} \rightarrow L^{2, \infty}$ boundedness of $M_{2}$, completes the proof.

Since $\|g\|_{L^{\infty}} \leq 2^{n} A \alpha$ and $\|g\|_{L^{1}} \leq\|f\|_{L^{1}}$, by Lemma 2.5.

$$
\begin{aligned}
\left|\left\{x: M_{\lambda / 2, T_{\Omega}}^{\mathcal{F}} g(x)>\alpha / 2\right\}\right| & \leq \frac{C_{n}}{\lambda \alpha^{2}}\left\|T_{\Omega}\right\|_{L^{2} \rightarrow L^{2}}^{2} \int_{\mathbb{R}^{n}}|g|^{2} d x \\
& \leq \frac{C_{n} A\left\|T_{\Omega}\right\|_{L^{2} \rightarrow L^{2}}^{2}}{\lambda} \frac{\|f\|_{L^{1}}}{\alpha}
\end{aligned}
$$

2.7. Estimate of the bad part. Pick $\psi \in C^{\infty}(\mathbb{R})$ such that $\operatorname{supp} \psi \subset$ $[1 / 2,2]$ and $\sum_{j \in \mathbb{Z}} \psi\left(2^{-j} t\right) \equiv 1$ for all $t \neq 0$. Denote $K(x)=\frac{\Omega(x /|x|)}{|x|^{n}}$, and set $K_{j}(x)=\psi\left(2^{-j}|x|\right) K(x)$ and $B_{l}=\sum_{|P|=2^{n l}} b_{P}(x)$. Then $K=$ $\sum_{j \in \mathbb{Z}} K_{j}$ and $b=\sum_{l \in \mathbb{Z}} B_{l}$.

Assume that $|P|=2^{n(j-s)}$ and $x \notin \gamma P, \gamma>1$. Since

$$
\operatorname{dist}\left((\gamma P)^{c}, P\right)=\frac{\gamma-1}{2} \ell_{P}=\frac{\gamma-1}{2} 2^{j-s},
$$


we obtain that if $2^{s}<\frac{\gamma-1}{4}$, then $\operatorname{dist}(x, P)>2^{j+1}$, and therefore $\left|K_{j}\right| *\left|b_{P}\right|(x)=0$. Setting in this argument $\gamma=9$, we conclude that for every cube $Q$,

$$
\operatorname{supp}\left(\sum_{s<1} \sum_{j \in \mathbb{Z}} K_{j} *\left(B_{j-s} \chi_{\mathbb{R}^{n} \backslash 3 Q}\right)\right) \subset \bigcup_{P \in \mathcal{P}} 9 P .
$$

Set now $E=\cup_{P \in \mathcal{P}} 9 P$ and $E^{*}=\left\{x: M^{\mathscr{D}} \chi_{E}(x)>\lambda / 8\right\}$. Observe that

$$
\begin{aligned}
\left|E^{*}\right| \leq \frac{8}{\lambda}|E| & \leq \frac{9^{n+1}}{\lambda}\left|\cup_{P \in \mathcal{P}} P\right| \\
& \leq \frac{9^{n+1}}{\lambda A} \frac{\|f\|_{L^{1}}}{\alpha} .
\end{aligned}
$$

Assume that $x \notin E^{*}$, and let $Q \in \mathcal{F}, x \in Q$. Then $|Q \cap E| \leq \frac{\lambda}{8}|Q|$, and hence, by (2.12),

$$
\left(\sum_{s<1} \sum_{j \in \mathbb{Z}} K_{j} *\left(B_{j-s} \chi_{\mathbb{R}^{n} \backslash 3 Q}\right) \chi_{Q}\right)^{*}(\lambda|Q| / 4)=0 .
$$

Therefore, using that

$$
T_{\Omega}\left(b \chi_{\mathbb{R}^{n} \backslash 3 Q}\right)(x)=\sum_{s \in \mathbb{Z}} \sum_{j \in \mathbb{Z}} K_{j} *\left(B_{j-s} \chi_{\mathbb{R}^{n} \backslash 3 Q}\right)(x),
$$

we obtain

$$
\begin{aligned}
& \left(T_{\Omega}\left(b \chi_{\mathbb{R}^{n} \backslash 3 Q}\right) \chi_{Q}\right)^{*}(\lambda|Q| / 2) \\
& \leq\left(\sum_{s \geq 1} \sum_{j \in \mathbb{Z}} K_{j} *\left(B_{j-s} \chi_{\mathbb{R}^{n} \backslash 3 Q}\right) \chi_{Q}\right)^{*}(\lambda|Q| / 4) .
\end{aligned}
$$

Let $m \in \mathbb{N}, m \geq 2$, that will be specified later. Set

$$
\mathcal{M}_{\lambda} b(x)=\max _{Q \ni x, Q \in \mathcal{F}}\left(\sum_{s \geq m} \sum_{j \in \mathbb{Z}} K_{j} *\left(B_{j-s} \chi_{\mathbb{R}^{n} \backslash 3 Q}\right) \chi_{Q}\right)^{*}(\lambda|Q| / 4)
$$

for $x \in \cup_{Q \in \mathcal{F}} Q$, and $\mathcal{M}_{\lambda} b(x)=0$ otherwise. Set also $T_{j} f(x)=K_{j} * f$. Then

$$
\begin{aligned}
& \left(\sum_{s \geq 1} \sum_{j \in \mathbb{Z}} K_{j} *\left(B_{j-s} \chi_{\mathbb{R}^{n} \backslash 3 Q}\right) \chi_{Q}\right)^{*}(\lambda|Q| / 4) \\
& \leq \sum_{s=1}^{m-1} \sum_{j \in \mathbb{Z}} M_{T_{j}}\left(B_{j-s}\right)(x)+\mathcal{M}_{\lambda} b(x),
\end{aligned}
$$

which, along with (2.14), yields

$$
M_{\lambda / 2, T_{\Omega}}^{\mathcal{F}} b(x) \leq \sum_{s=1}^{m-1} \sum_{j \in \mathbb{Z}} M_{T_{j}}\left(B_{j-s}\right)(x)+\mathcal{M}_{\lambda} b(x) \quad\left(x \notin E^{*}\right) .
$$


Therefore,

$$
\begin{aligned}
& \left|\left\{x: M_{\lambda / 2, T_{\Omega}}^{\mathcal{F}} b(x)>\alpha / 2\right\}\right| \leq\left|E^{*}\right| \\
& +\frac{4}{\alpha} \sum_{s=1}^{m-1} \sum_{j \in \mathbb{Z}}\left\|M_{T_{j}}\left(B_{j-s}\right)\right\|_{L^{1}}+\left|\left\{x: \mathcal{M}_{\lambda} b(x)>\alpha / 4\right\}\right| .
\end{aligned}
$$

\subsection{Estimate of $M_{T_{j}}$.}

Lemma 2.6. The operator $M_{T_{j}}$ is $L^{1}$ bounded, and

$$
\left\|M_{T_{j}}(f)\right\|_{L^{1}} \leq C_{n}\|\Omega\|_{L^{\infty}}\|f\|_{L^{1}}
$$

Proof. Let $x, \xi \in Q$. If $\ell_{Q}>2^{j-1}$, then $\xi-y \notin \operatorname{supp}\left(K_{j}\right)$ for every $y \in \mathbb{R}^{n} \backslash 3 Q$, and hence

$$
K_{j} *\left(f \chi_{\mathbb{R}^{n} \backslash 3 Q}\right)(\xi)=0 .
$$

Assume that $\ell_{Q} \leq 2^{j-1}$. Suppose also that $y \in \mathbb{R}^{n} \backslash 3 Q$ and $|y-\xi| \leq$ $2^{j+1}$. Then

$$
|y-x| \leq|y-\xi|+|\xi-x| \leq 2^{j+1}+\sqrt{n} \ell_{Q} \leq 2^{j}(2+\sqrt{n} / 2),
$$

and hence,

$$
\begin{aligned}
\left|K_{j}(\xi-y)\right| & \leq \frac{\|\Omega\|_{L^{\infty}}}{2^{(j-1) n}} \chi_{\left\{|y-\xi| \leq 2^{j+1}\right\}}(y) \\
& \leq \frac{\|\Omega\|_{L^{\infty}}}{2^{(j-1) n}} \chi_{\left\{|y-x| \leq 2^{j}(2+\sqrt{n} / 2)\right\}}(y) .
\end{aligned}
$$

Therefore,

$$
\left|K_{j} *\left(f \chi_{\mathbb{R}^{n} \backslash 3 Q}\right)(\xi)\right| \leq \frac{\|\Omega\|_{L^{\infty}}}{2^{(j-1) n}} \int_{\left\{|y-x| \leq 2^{j}(2+\sqrt{n} / 2)\right\}}|f(y)| d y
$$

Thus,

$$
M_{T_{j}}(f)(x) \leq \frac{\|\Omega\|_{L^{\infty}}}{2^{(j-1) n}} \int_{\left\{|y-x| \leq 2^{j}(2+\sqrt{n} / 2)\right\}}|f(y)| d y,
$$

which implies (2.16).

Applying Lemma 2.6 yields

$$
\begin{aligned}
\sum_{s=1}^{m-1} \sum_{j \in \mathbb{Z}}\left\|M_{T_{j}}\left(B_{j-s}\right)\right\|_{L^{1}} & \leq C_{n}\|\Omega\|_{L^{\infty}} \sum_{s=1}^{m-1} \sum_{j \in \mathbb{Z}}\left\|B_{j-s}\right\|_{L^{1}} \\
& \leq C_{n} m\|\Omega\|_{L^{\infty}}\|f\|_{L^{1}}
\end{aligned}
$$


2.9. Estimate of $\mathcal{M}_{\lambda} b$. Write the set $\left\{x: \mathcal{M}_{\lambda} b(x)>\alpha / 4\right\}$ as the union of the maximal pairwise disjoint cubes $Q_{i} \in \mathcal{F}$ with the property

$$
\left(\sum_{s \geq m} \sum_{j \in \mathbb{Z}} K_{j} *\left(B_{j-s} \chi_{\mathbb{R}^{n} \backslash 3 Q_{i}}\right) \chi_{Q_{i}}\right)^{*}\left(\lambda\left|Q_{i}\right| / 4\right)>\alpha / 4,
$$

or, equivalently,

$$
\left|Q_{i}\right|<\frac{4}{\lambda}\left|\left\{x \in Q_{i}:\left|\sum_{s \geq m} \sum_{j \in \mathbb{Z}} K_{j} *\left(B_{j-s} \chi_{\mathbb{R}^{n} \backslash 3 Q_{i}}\right)(x)\right|>\alpha / 4\right\}\right| .
$$

It follows that the cubes $Q_{i}$ can be selected into two disjoint families: let $\mathcal{A}_{1}$ be the family of $Q_{i}$ for which

$$
\left|Q_{i}\right|<\frac{8}{\lambda}\left|\left\{x \in Q_{i}:\left|\sum_{s \geq m} \sum_{j \in \mathbb{Z}} K_{j} * B_{j-s}(x)\right|>\alpha / 8\right\}\right|,
$$

and let $\mathcal{A}_{2}$ be the family of $Q_{i}$ for which

$$
\left|Q_{i}\right|<\frac{8}{\lambda}\left|\left\{x \in Q_{i}:\left|\sum_{s \geq m} \sum_{j \in \mathbb{Z}} K_{j} *\left(B_{j-s} \chi_{3 Q_{i}}\right)(x)\right|>\alpha / 8\right\}\right| .
$$

2.10. The cubes from the first family. We have

$$
\sum_{Q_{i} \in \mathcal{A}_{1}}\left|Q_{i}\right|<\frac{8}{\lambda}\left|\left\{x \in \mathbb{R}^{n}:\left|\sum_{s \geq m} \sum_{j \in \mathbb{Z}} K_{j} * B_{j-s}(x)\right|>\alpha / 8\right\}\right| .
$$

To estimate the right-hand side here, we use the following result by Seeger [17] (in the next statement we unified Lemmata 2.1 and 2.2 from [17]).

Lemma 2.7. Let $\left\{H_{j}\right\}$ be a family of functions supported in $\left\{x: 2^{j-2} \leq\right.$ $\left.|x| \leq 2^{j+2}\right\}$ and such that the estimates

$$
\sup _{0 \leq l \leq N} \sup _{j} r^{n+l}\left|\left(\frac{\partial}{\partial r}\right)^{l} H_{j}(r \theta)\right| \leq M_{N}
$$

hold uniformly in $\theta \in S^{n-1}$ and $r>0$. Then for every $0<\kappa<1$ and any natural $s>3$ one can split $H_{j}=\Gamma_{j}^{s}+\left(H_{j}-\Gamma_{j}^{s}\right)$ such that the following properties hold.

(1) Let $\mathcal{Q}$ be a collection of pairwise disjoint dyadic cubes, and let $\mathcal{Q}_{m}=\left\{Q \in \mathcal{Q}:|Q|=2^{n m}\right\}, m \in \mathbb{Z}$. For each $Q \in \mathcal{Q}$ let $f_{Q}$ be an integrable function supported in $Q$ satisfying $\int\left|f_{Q}\right| d x \leq$ $\alpha|Q|$. Let $F_{m}=\sum_{Q \in \mathcal{Q}_{m}} f_{Q}$. Then for $s>3$,

$$
\left\|\sum_{j} \Gamma_{j}^{s} * F_{j-s}\right\|_{L^{2}}^{2} \leq C_{n} M_{0}^{2} 2^{-s(1-\kappa)} \alpha \sum_{Q}\left\|f_{Q}\right\|_{L^{1}}
$$


(2) Let $Q$ be a cube of sidelength $2^{j-s}$ and let $b_{Q}$ be integrable and supported in $Q$ with $\int_{Q} b_{Q}=0$. Then for $N \geq n+1$ and $0 \leq \varepsilon \leq 1$

$$
\left\|\left(H_{j}-\Gamma_{j}^{s}\right) * b_{Q}\right\|_{L^{1}} \leq C_{n, N}\left(M_{0} 2^{-s \varepsilon}+M_{N} 2^{s(n+(\varepsilon-\kappa) N)}\right)\left\|b_{Q}\right\|_{L^{1}} .
$$

Notice that $K_{j}$ is supported in $\left\{2^{j-1} \leq|x| \leq 2^{j+1}\right\}$ and

$$
\sup _{0 \leq l \leq N} \sup _{j} r^{n+l}\left|\left(\frac{\partial}{\partial r}\right)^{l} K_{j}(r \theta)\right| \leq C_{N, n}\|\Omega\|_{L^{\infty}} .
$$

Therefore, we are in position to apply Lemma 2.7. Choose in this lemma $\kappa=\frac{1}{2}$ and $\varepsilon=\frac{1}{4}$. We obtain

$$
\begin{aligned}
& \left|\left\{x:\left|\sum_{s \geq m} \sum_{j \in \mathbb{Z}} K_{j} * B_{j-s}\right|>\alpha / 8\right\}\right| \\
& \leq|\{x:|I(x)|>\alpha / 16\}|+|\{x:|I I(x)|>\alpha / 16\}|,
\end{aligned}
$$

where

$$
I(x)=\sum_{s \geq m} \sum_{j \in \mathbb{Z}} \Gamma_{j}^{s} * B_{j-s}(x)
$$

and

$$
I I(x)=\sum_{s \geq m} \sum_{j \in \mathbb{Z}}\left(K_{j}-\Gamma_{j}^{s}\right) * B_{j-s}(x) .
$$

Observe that

$$
\int_{P}\left|b_{P}\right| d x \leq 2 \int_{P}|f| \leq 2^{n+1} A \alpha|P| .
$$

Therefore, the first part of Lemma 2.7 yields

$$
\begin{aligned}
& |\{x:|I(x)|>\alpha / 16\}| \leq \frac{256}{\alpha^{2}}\|I\|_{L^{2}}^{2} \leq \frac{256}{\alpha^{2}}\left(\sum_{s \geq m}\left\|\sum_{j} \Gamma_{j}^{s} * B_{j-s}\right\|_{L^{2}}\right)^{2} \\
& \leq \frac{C_{n}\|\Omega\|_{L^{\infty}}^{2}}{\alpha^{2}}\left(\sum_{s \geq m} 2^{-s / 4}\left(A \alpha \sum_{P}\left\|b_{P}\right\|_{L^{1}}\right)^{1 / 2}\right)^{2} \\
& \leq C_{n}\|\Omega\|_{L^{\infty}}^{2} \frac{A 2^{-m / 2}}{\alpha}\|f\|_{L^{1}} .
\end{aligned}
$$

Applying the second part of Lemma 2.7 with $N=8 n$ yields,

$$
\begin{aligned}
& |\{x:|I I(x)|>\alpha / 16\}| \leq \frac{16}{\alpha} \sum_{s \geq m}\left\|\sum_{j \in \mathbb{Z}}\left(K_{j}-\Gamma_{j}^{s}\right) * B_{j-s}\right\|_{L^{1}} \\
& \leq \frac{C_{n}\|\Omega\|_{L^{\infty}}}{\alpha} \sum_{s \geq m}\left(2^{-s / 4}+2^{-n s}\right)\|f\|_{L^{1}} \leq C_{n}\|\Omega\|_{L^{\infty}} 2^{-m / 4} \frac{\|f\|_{L^{1}}}{\alpha} .
\end{aligned}
$$


Combining the estimates for $I$ and $I I$ with (2.18) and (2.19), we obtain

$$
\sum_{Q_{i} \in \mathcal{A}_{1}}\left|Q_{i}\right| \leq \frac{C_{n}}{\lambda}\left(\|\Omega\|_{L^{\infty}}^{2} A 2^{-m / 2}+\|\Omega\|_{L^{\infty}} 2^{-m / 4}\right) \frac{\|f\|_{L^{1}}}{\alpha}
$$

2.11. The cubes from the second family. Let $Q_{i} \in \mathcal{A}_{2}$. Observe that the cube $3 Q_{i}$ and the cubes appearing in the definition of $B_{j-s}$ are from the same dyadic lattice $\mathscr{D}^{\prime}$. Therefore, setting

$$
B_{j-s}^{(i)}=\sum_{P:|P|=2^{(j-s) n}, P \subset 3 Q_{i}} b_{P}(x),
$$

we obtain that for $2^{j-s}<3 \ell_{Q_{i}}$,

$$
B_{j-s} \chi_{3 Q_{i}}=B_{j-s}^{(i)} .
$$

Assume that $2^{j-s} \geq 3 \ell_{Q_{i}}$. Then for all $x \in Q_{i}$ and $y \in 3 Q_{i}$,

$$
|x-y| \leq 2 \sqrt{n} \ell_{Q_{i}} \leq \frac{4 \sqrt{n}}{3} \frac{1}{2^{s}} 2^{j-1},
$$

and therefore, $x-y \notin \operatorname{supp} K_{j}$, provided $2^{s}>4 \sqrt{n} / 3$. Hence, assuming that $m$ is such that $2^{m}>4 \sqrt{n} / 3$, for all $s \geq m$ we obtain

$$
K_{j} *\left(B_{j-s} \chi_{3 Q_{i}}\right)(x)=0,
$$

which implies

$$
\begin{aligned}
\left|Q_{i}\right| & <\frac{8}{\lambda}\left|\left\{x \in Q_{i}:\left|\sum_{s \geq m} \sum_{j \in \mathbb{Z}} K_{j} *\left(B_{j-s} \chi_{3 Q_{i}}\right)(x)\right|>\alpha / 8\right\}\right| \\
& \leq \frac{8}{\lambda}\left|\left\{x:\left|\sum_{s \geq m} \sum_{j \in \mathbb{Z}} K_{j} * B_{j-s}^{(i)}(x)\right|>\alpha / 8\right\}\right| .
\end{aligned}
$$

Denote by $\gamma$ the constant appearing on the right-hand side of (2.20), that is, let

$$
\gamma=\frac{C_{n}}{\lambda \alpha}\left(\|\Omega\|_{L^{\infty}}^{2} A 2^{-m / 2}+\|\Omega\|_{L^{\infty}} 2^{-m / 4}\right) .
$$

Then, arguing exactly as in the proof of (2.20) and using that all the cubes in the definition of $B_{j-s}^{(i)}$ are supported in $3 Q_{i}$, we obtain

$$
\left|Q_{i}\right| \leq \gamma \int_{3 Q_{i}}|f|
$$

Hence,

$$
\cup_{Q_{i} \in \mathcal{A}_{2}} Q_{i} \subset\left\{x: M f(x)>1 /\left(3^{n} \gamma\right)\right\},
$$


which implies that the cubes from the second family satisfy the same estimate as (2.20). Therefore,

$$
\left|\left\{x: \mathcal{M}_{\lambda} b(x)>\alpha / 4\right\}\right| \leq \frac{C_{n}}{\lambda}\left(\|\Omega\|_{L^{\infty}}^{2} A 2^{-m / 2}+\|\Omega\|_{L^{\infty}} 2^{-m / 4}\right) \frac{\|f\|_{L^{1}}}{\alpha} .
$$

2.12. Conclusion of the proof. Assume that $2^{m}>4 \sqrt{n} / 3$. Combining the last estimate with (2.10), (2.11), (2.13), (2.15) and (2.17) yields

$$
\begin{aligned}
& \left\|M_{\lambda, T_{\Omega}}\right\|_{L^{1} \rightarrow L^{1, \infty}} \leq C_{n}\left(\|\Omega\|_{L^{\infty}} m\right. \\
& \left.+\frac{1}{\lambda}\left(A\left\|T_{\Omega}\right\|_{L^{2} \rightarrow L^{2}}^{2}+\frac{9^{n+1}}{A}+\|\Omega\|_{L^{\infty}}^{2} A 2^{-m / 2}+\|\Omega\|_{L^{\infty}} 2^{-m / 4}\right)\right) .
\end{aligned}
$$

From this, setting $\nu=\max \left(\|\Omega\|_{L^{\infty}},\left\|T_{\Omega}\right\|_{L^{2} \rightarrow L^{2}} / \delta\right)$, where $0<\delta \leq 1$, and $A=\frac{2^{m / 4}}{\nu}$, we obtain

$$
\left\|M_{\lambda, T_{\Omega}}\right\|_{L^{1} \rightarrow L^{1, \infty}} \leq C_{n} \nu\left(\frac{1}{\lambda}\left(2^{m / 4} \delta^{2}+2^{-m / 4}\right)+m\right) .
$$

One can assume that $\delta^{-4}>4 \sqrt{n} / 3$ since otherwise Lemma 2.3 is trivial. Then, set in (2.21) $m \in \mathbb{N}$ such that $2^{m-1} \leq \delta^{-4}<2^{m}$. We obtain

$$
\left\|M_{\lambda, T_{\Omega}}\right\|_{L^{1} \rightarrow L^{1, \infty}} \leq C_{n} \nu\left(\frac{\delta}{\lambda}+\log \frac{2}{\delta}\right)
$$

which completes the proof.

\section{A SPARSE DOMINATION PRINCIPLE}

We start with the following general result which can be described in terms of the bi-sublinear maximal operator $\mathscr{M}_{T}$ defined for a given operator $T$ by

$$
\mathscr{M}_{T}(f, g)(x)=\sup _{Q \ni x} \frac{1}{|Q|} \int_{Q}\left|T\left(f \chi_{\mathbb{R}^{n} \backslash 3 Q}\right)\right||g| d y,
$$

where the supremum is taken over all cubes $Q \subset \mathbb{R}^{n}$ containing $x$.

Theorem 3.1. Let $1 \leq q \leq r$ and $s \geq 1$. Assume that $T$ is a sublinear operator of weak type $(q, q)$, and $\mathscr{M}_{T}$ maps $L^{r} \times L^{s}$ into $L^{\nu, \infty}$, where $\frac{1}{\nu}=\frac{1}{r}+\frac{1}{s}$. Then, for every compactly supported $f \in L^{r}\left(\mathbb{R}^{n}\right)$ and every $g \in L_{\text {loc }}^{s}$, there exists a $\frac{1}{2 \cdot 3^{n}}$-sparse family $\mathcal{S}$ such that

$$
|\langle T f, g\rangle| \leq K \sum_{Q \in \mathcal{S}}\langle f\rangle_{r, Q}\langle g\rangle_{s, Q}|Q|
$$

where

$$
K=C_{n}\left(\|T\|_{L^{q} \rightarrow L^{q, \infty}}+\left\|\mathscr{M}_{T}\right\|_{L^{r} \times L^{s} \rightarrow L^{\nu, \infty}}\right) .
$$


Proof. The proof is very similar to the one of [15, Th. 4.2].

Fix a cube $Q_{0}$. Define a local analogue of $\mathscr{M}_{T}$ by

$$
\mathscr{M}_{T, Q_{0}}(f, g)(x)=\sup _{Q \ni x, Q \subset Q_{0}} \frac{1}{|Q|} \int_{Q}\left|T\left(f \chi_{3 Q_{0} \backslash 3 Q}\right)\right||g| d y .
$$

Consider the sets

$$
E_{1}=\left\{x \in Q_{0}:\left|T\left(f \chi_{3 Q_{0}}\right)(x)\right|>A\langle f\rangle_{q, 3 Q_{0}}\right\}
$$

and

$$
E_{2}=\left\{x \in Q_{0}: \mathscr{M}_{T, Q_{0}}(f, g)(x)>B\langle f\rangle_{r, 3 Q_{0}}\langle g\rangle_{s, Q_{0}}\right\},
$$

where $A$ and $B$ are chosen in such a way that

$$
\max \left(\left|E_{1}\right|,\left|E_{2}\right|\right) \leq \frac{1}{2^{n+3}}\left|Q_{0}\right|
$$

namely, we take

$$
A=\left(8 \cdot 6^{n}\right)^{1 / q}\|T\|_{L^{q} \rightarrow L^{q, \infty}} \quad \text { and } \quad B=\left(2^{n+3}\right)^{1 / \nu} 3^{n / r}\left\|\mathscr{M}_{T}\right\|_{L^{r} \times L^{s} \rightarrow L^{\nu, \infty}} .
$$

Then, the set $\Omega=E_{1} \cup E_{2}$ satisfies $|\Omega| \leq \frac{1}{2^{n+2}}\left|Q_{0}\right|$.

The Calderón-Zygmund decomposition applied to the function $\chi_{\Omega}$ on $Q_{0}$ at height $\lambda=\frac{1}{2^{n+1}}$ produces pairwise disjoint cubes $P_{j} \in \mathcal{D}\left(Q_{0}\right)$ such that

$$
\frac{1}{2^{n+1}}\left|P_{j}\right| \leq\left|P_{j} \cap E\right| \leq \frac{1}{2}\left|P_{j}\right|
$$

and $\left|\Omega \backslash \cup_{j} P_{j}\right|=0$. It follows that $\sum_{j}\left|P_{j}\right| \leq \frac{1}{2}\left|Q_{0}\right|$ and $P_{j} \cap \Omega^{c} \neq \emptyset$.

Since $\left|\Omega \backslash \cup_{j} P_{j}\right|=0$, we have

$$
\int_{Q_{0} \backslash \cup_{j} P_{j}}\left|T\left(f \chi_{3 Q_{0}}\right)\right||g| \leq A\langle f\rangle_{q, 3 Q_{0}} \int_{Q_{0}}|g| .
$$

On the other hand, since $P_{j} \cap \Omega^{c} \neq \emptyset$, we obtain

$$
\int_{P_{j}}\left|T\left(f \chi_{3 Q_{0} \backslash 3 P_{j}}\right)\right||g| \leq B\langle f\rangle_{r, 3 Q_{0}}\langle g\rangle_{s, Q_{0}}\left|P_{j}\right| .
$$

Combining these estimates along with Hölder's inequality (here we use that $q \leq r$ and $s \geq 1$ ) yields

$$
\begin{aligned}
& \int_{Q_{0}}\left|T\left(f \chi_{3 Q_{0}}\right)\right||g| \leq \int_{Q_{0} \backslash \cup_{j} P_{j}}\left|T\left(f \chi_{3 Q_{0}}\right)\right||g| \\
& +\sum_{j} \int_{P_{j}}\left|T\left(f \chi_{3 Q_{0} \backslash 3 P_{j}}\right)\right||g|+\sum_{j} \int_{P_{j}}\left|T\left(f \chi_{3 P_{j}}\right)\right||g| \\
& \leq(A+B)\langle f\rangle_{r, 3 Q_{0}}\langle g\rangle_{s, Q_{0}}\left|Q_{0}\right|+\sum_{j} \int_{P_{j}}\left|T\left(f \chi_{3 P_{j}}\right)\right||g| .
\end{aligned}
$$


Since $\sum_{j}\left|P_{j}\right| \leq \frac{1}{2}\left|Q_{0}\right|$, iterating the above estimate, we obtain that there is a $\frac{1}{2}$-sparse family $\mathcal{F} \subset \mathcal{D}\left(Q_{0}\right)$ such that

$$
\int_{Q_{0}}\left|T\left(f \chi_{3 Q_{0}}\right)\right||g| \leq \sum_{Q \in \mathcal{F}}(A+B)\langle f\rangle_{r, 3 Q}\langle g\rangle_{s, Q}|Q|
$$

(notice that $\mathcal{F}=\left\{P_{j}^{k}\right\}, k \in \mathbb{Z}_{+}$, where $\left\{P_{j}^{0}\right\}=\left\{Q_{0}\right\},\left\{P_{j}^{1}\right\}=\left\{P_{j}\right\}$ and $\left\{P_{j}^{k}\right\}$ are the cubes obtained at the $k$-th stage of the iterative process).

Take now a partition of $\mathbb{R}^{n}$ by cubes $R_{j}$ such that $\operatorname{supp}(f) \subset 3 R_{j}$ for each $j$. For example, take a cube $Q_{0}$ such that $\operatorname{supp}(f) \subset Q_{0}$ and cover $3 Q_{0} \backslash Q_{0}$ by $3^{n}-1$ congruent cubes $R_{j}$. Each of them satisfies $Q_{0} \subset 3 R_{j}$. Next, in the same way cover $9 Q_{0} \backslash 3 Q_{0}$, and so on. The union of resulting cubes, including $Q_{0}$, will satisfy the desired property.

Having such a partition, apply (3.2) to each $R_{j}$. We obtain a $\frac{1}{2}$-sparse family $\mathcal{F}_{j} \subset \mathcal{D}\left(R_{j}\right)$ such that

$$
\int_{R_{j}}|T(f)||g| \leq \sum_{Q \in \mathcal{F}_{j}}(A+B)\langle f\rangle_{r, 3 Q}\langle g\rangle_{s, Q}|Q|
$$

Therefore,

$$
\int_{\mathbb{R}^{n}}|T(f)||g| \leq \sum_{Q \in \cup_{j} \mathcal{F}_{j}}(A+B)\langle f\rangle_{r, 3 Q}\langle g\rangle_{s, Q}|Q|
$$

Notice that the family $\cup_{j} \mathcal{F}_{j}$ is $\frac{1}{2}$-sparse as a disjoint union of $\frac{1}{2}$-sparse families. Hence, setting $\mathcal{S}=\left\{3 Q: Q \in \cup_{j} \mathcal{F}_{j}\right\}$, we obtain that $\mathcal{S}$ is $\frac{1}{2 \cdot 3^{n}}$-sparse, and (3.1) holds.

Given $1 \leq p \leq \infty$, define the maximal operator $\mathscr{M}_{p, T}$ by

$$
\mathscr{M}_{p, T} f(x)=\sup _{Q \ni x}\left(\frac{1}{|Q|} \int_{Q}\left|T\left(f \chi_{\mathbb{R}^{n} \backslash 3 Q}\right)\right|^{p} d y\right)^{1 / p}
$$

(in the case $p=\infty$ set $\mathscr{M}_{p, T} f(x)=M_{T} f(x)$ ).

Corollary 3.2. Let $1 \leq q \leq r$ and $s \geq 1$. Assume that $T$ is a sublinear operator of weak type $(q, q)$, and $\mathscr{M}_{s^{\prime}, T}$ is of weak type $(r, r)$. Then, for every compactly supported $f \in L^{r}\left(\mathbb{R}^{n}\right)$ and every $g \in L_{\text {loc }}^{s}$, there exists a $\frac{1}{2 \cdot 3^{n}}$-sparse family $\mathcal{S}$ such that

$$
|\langle T f, g\rangle| \leq K \sum_{Q \in \mathcal{S}}\langle f\rangle_{r, Q}\langle g\rangle_{s, Q}|Q|
$$

where

$$
K=C_{n}\left(\|T\|_{L^{q} \rightarrow L^{q, \infty}}+\left\|\mathscr{M}_{s^{\prime}, T}\right\|_{L^{r} \rightarrow L^{r, \infty}}\right)
$$


Proof. By Hölder's inequality,

$$
\mathscr{M}_{T}(f, g)(x) \leq \mathscr{M}_{s^{\prime}, T} f(x) M_{s} g(x),
$$

where $M_{s} g=M\left(g^{s}\right)^{1 / s}$. From this, by Hölder's inequality for weak spaces (see [9, p. 15]) along with the weak type $(s, s)$ estimate for $M_{s}$,

$$
\left\|\mathscr{M}_{T}\right\|_{L^{r} \times L^{s} \rightarrow L^{\nu, \infty}} \leq C_{n}\left\|\mathscr{M}_{s^{\prime}, T}\right\|_{L^{r} \rightarrow L^{r, \infty}} \quad(1 / \nu=1 / r+1 / s),
$$

which, by Theorem 3.1, completes the proof.

In order to apply Corollary 3.2 to Theorem 1.1, we first establish a relation between the $L^{1} \rightarrow L^{1, \infty}$ norms of the operators $M_{\lambda, T}$ and $\mathscr{M}_{p, T}$.

Lemma 3.3. Let $0<\gamma \leq 1$ and let $T$ be a sublinear operator. The following statements are equivalent:

(i) there exists $C>0$ such that for all $p \geq 1$,

$$
\left\|\mathscr{M}_{p, T} f\right\|_{L^{1} \rightarrow L^{1, \infty}} \leq C p^{\gamma}
$$

(ii) there exists $C>0$ such that for all $0<\lambda<1$,

$$
\left\|M_{\lambda, T} f\right\|_{L^{1} \rightarrow L^{1, \infty}} \leq C\left(1+\log \frac{1}{\lambda}\right)^{\gamma} .
$$

Proof. Let us show that (i) $\Rightarrow($ ii). By Chebyshev's inequality,

$$
M_{\lambda, T} f(x) \leq \frac{1}{\lambda^{1 / p}} \mathscr{M}_{p, T} f(x),
$$

which implies

$$
\left\|M_{\lambda, T} f\right\|_{L^{1, \infty}} \leq \frac{1}{\lambda^{1 / p}}\left\|\mathscr{M}_{p, T} f\right\|_{L^{1, \infty}} \leq C \frac{p^{\gamma}}{\lambda^{1 / p}}\|f\|_{L^{1}} .
$$

Setting here $p=\max \left(1, \log \frac{1}{\lambda}\right)$, we obtain (ii).

Turn to the implication (ii) $\Rightarrow(\mathrm{i})$. First, observe that

$$
\left(\frac{1}{|Q|} \int_{Q}\left|T\left(f \chi_{\mathbb{R}^{n} \backslash 3 Q}\right)\right|^{p} d y\right)^{1 / p}=\left(\int_{0}^{1}\left(T\left(f \chi_{\mathbb{R}^{n} \backslash 3 Q}\right) \chi_{Q}\right)^{*}(\lambda|Q|)^{p} d \lambda\right)^{1 / p}
$$

which implies

$$
\mathscr{M}_{p, T} f(x) \leq\left(\int_{0}^{1} M_{\lambda, T} f(x)^{p} d \lambda\right)^{1 / p} .
$$

For $N>0$ denote

$$
G_{p, T, N} f(x)=\left(\int_{0}^{1} \min \left(M_{\lambda, T} f(x), N\right)^{p} d \lambda\right)^{1 / p} .
$$

Set also $\mu_{f}(\alpha, R)=|\{|x| \leq R:|f(x)|>\alpha\}|$ for $R, \alpha>0$. 
Let $k>1$ that will be specified later. By Hölder's inequality,

$$
\begin{aligned}
G_{p, T, N} f(x) & \leq\left(\int_{0}^{1 / 2^{k p}} \min \left(M_{\lambda, T} f(x), N\right)^{p} d \lambda\right)^{1 / p}+M_{1 / 2^{k p}, T} f(x) \\
& \leq \frac{1}{2^{k-1}} G_{k p, T, N} f(x)+M_{1 / 2^{k p}, T} f(x) .
\end{aligned}
$$

Hence,

$$
\begin{aligned}
\mu_{G_{p, T, N} f}(\alpha, R) & \leq \mu_{G_{k p, T, N} f}\left(2^{k-2} \alpha, R\right)+\mu_{M_{1 / 2^{k p}, T} f}(\alpha / 2, R) \\
& \leq \mu_{G_{k p, T, N} f}\left(2^{k-2} \alpha, R\right)+C \frac{(k p)^{\gamma}}{\alpha}\|f\|_{L^{1}} .
\end{aligned}
$$

Iterating this estimate, after the $j$-th step we obtain

$$
\mu_{G_{p, T, N} f}(\alpha, R) \leq \mu_{G_{k^{j}, T, N} f}\left(2^{(k-2) j} \alpha, R\right)+\frac{2^{k-2} C}{\alpha} \sum_{i=1}^{j}\left(\frac{k^{\gamma}}{2^{k-2}}\right)^{i} p^{\gamma}\|f\|_{L^{1}} .
$$

Take here $k=5$. Since $G_{p, T, N}$ is bounded uniformly in $p$, we obtain that $\mu_{G_{5 j}{ }_{p, T, N} f}\left(8^{j} \alpha, R\right)=0$ starting from some $j$ big enough. Hence, letting $j \rightarrow \infty$ in the above estimate yields

$$
\mu_{G_{p, T, N} f}(\alpha, R) \leq \frac{C}{\alpha} \sum_{i=1}^{\infty}\left(\frac{5}{8}\right)^{i} p^{\gamma}\|f\|_{L^{1}} \leq \frac{C^{\prime}}{\alpha} p^{\gamma}\|f\|_{L^{1}}
$$

Letting here $N, R \rightarrow \infty$ and applying (3.3) completes the proof.

Now, we are ready to show that Theorem 1.1 provides a different approach to (1.1).

Corollary 3.4. Let $T_{\Omega}$ be a rough homogeneous singular integral with $\Omega \in L^{\infty}\left(S^{n-1}\right)$. Then, for every compactly supported $f \in L^{p}\left(\mathbb{R}^{n}\right)$ and every $g \in L_{\text {loc }}^{1}$, there exists a $\frac{1}{2 \cdot 3^{n}}$-sparse family $\mathcal{S}$ such that

$$
\left|\left\langle T_{\Omega} f, g\right\rangle\right| \leq C_{n} p^{\prime}\|\Omega\|_{L^{\infty}\left(S^{n-1}\right)} \sum_{Q \in \mathcal{S}}\langle f\rangle_{p, Q}\langle g\rangle_{1, Q}|Q| \quad(p>1) .
$$

Proof. By Theorem 1.1 along with Lemma 3.3 with $\gamma=1$,

$$
\left\|\mathscr{M}_{p, T_{\Omega}}\right\|_{L^{1} \rightarrow L^{1, \infty}} \leq C_{n}\|\Omega\|_{L^{\infty}\left(S^{n-1}\right)} p \quad(p \geq 1) .
$$

Also, by [17], $\left\|T_{\Omega}\right\|_{L^{1} \rightarrow L^{1, \infty}} \leq C_{n}\|\Omega\|_{L^{\infty}\left(S^{n-1}\right)}$. Hence, by Corollary 3.2 with $q=r=1$ and $s=p$, there exists a $\frac{1}{2 \cdot 3^{n}}$-sparse family $\mathcal{S}$ such that

$$
\left|\left\langle T_{\Omega} f, g\right\rangle\right| \leq C_{n} p^{\prime}\|\Omega\|_{L^{\infty}\left(S^{n-1}\right)} \sum_{Q \in \mathcal{S}}\langle f\rangle_{1, Q}\langle g\rangle_{p, Q}|Q| \quad(p>1) .
$$

Since the operator $T_{\Omega}$ is essentially self-adjoint, the same estimate holds for the adjoint operator $T_{\Omega}^{*}$. Replacing in the above estimate $T_{\Omega}$ by $T_{\Omega}^{*}$ and interchanging $f$ and $g$ completes the proof. 
Remark 3.5. Theorem 3.1 and Corollary 3.2 can be easily generalized by means of replacing the normalized $L^{p}$ averages by the normalized Orlicz averages $\|f\|_{\varphi, Q}$ defined by

$$
\|f\|_{\varphi, Q}=\inf \left\{\alpha>0: \frac{1}{|Q|} \int_{Q} \varphi(|f(y)| / \alpha) d y \leq 1\right\} .
$$

We mention only one interesting particular case of such a generalization. Denote $\|f\|_{L \log L, Q}$ if $\varphi(t)=t \log (\mathrm{e}+t)$ and $\|f\|_{\exp L, Q}$ if $\varphi(t)=e^{t}-1$. Given an operator $T$ define the maximal operator $M_{\exp L, T}$ by

$$
M_{\exp L, T} f(x)=\sup _{Q \ni x}\left\|T\left(f \chi_{\mathbb{R}^{n} \backslash 3 Q}\right)\right\|_{\exp L, Q} .
$$

Then if $T$ and $M_{\exp L, T}$ are of weak type $(1,1)$, for every appropriate $f$ and $g$, there exists a sparse family $\mathcal{S}$ such that

$$
|\langle T f, g\rangle| \leq K \sum_{Q \in \mathcal{S}}\langle f\rangle_{1, Q}\|g\|_{L \log L, Q}|Q|,
$$

where $K=C_{n}\left(\|T\|_{L^{1} \rightarrow L^{1, \infty}}+\left\|M_{\exp L, T}\right\|_{L^{1} \rightarrow L^{1, \infty}}\right)$.

In particular, we conjecture that if $T_{\Omega}$ is a rough homogeneous singular integral with $\Omega \in L^{\infty}\left(S^{n-1}\right)$, then $M_{\exp L, T_{\Omega}}$ is of weak type $(1,1)$. This would imply a small improvement of (1.1) with $p^{\prime}\langle f\rangle_{p, Q}, p>1$, replaced by $\|f\|_{L \log L, Q}$.

\section{REFERENCES}

[1] F. Bernicot, D. Frey and S. Petermichl, Sharp weighted norm estimates beyond Calderón-Zygmund theory, Anal. PDE 9 (2016), no. 5, 1079-1113.

[2] A.P. Calderón and A. Zygmund, On singular integrals, Amer. J. Math. 78 (1956), 289-309.

[3] M. Christ, Weak type $(1,1)$ bounds for rough operators, Ann. of Math. 128 (1988), no. 1, 19-42.

[4] M. Christ and J.L. Rubio de Francia, Weak type $(1,1)$ bounds for rough operators. II, Invent. Math. 93 (1988), no. 1, 225-237.

[5] J.M. Conde-Alonso, A. Culiuc, F. Di Plinio and Y. Ou, A sparse domination principle for rough singular integrals, preprint. Available at https://arxiv.org/abs/1612.09201, to appear in Anal. PDE.

[6] A. Culiuc, F. Di Plinio and Y. Ou, Domination of multilinear singular integrals by positive sparse forms, preprint. Available at https://arxiv.org/abs/1603.05317

[7] F. Di Plinio, Y.Q. Do and G.N. Uraltsev, Positive sparse domination of variational Carleson operators, preprint. Available at https://arxiv.org/abs/1612.03028, to appear in Ann. Sci. Scuola Norm. Sup. (Scienze).

[8] J. Duoandikoetxea and J.L. Rubio de Francia, Maximal and singular integral operators via Fourier transform estimates, Invent. Math., 84 (1986), no. 3, $541-561$. 
[9] L. Grafakos, Classical Fourier analysis. Second edition. Graduate Texts in Mathematics, 249. Springer, New York, 2009.

[10] S. Hofmann, Weak $(1,1)$ boundedness of singular integrals with nonsmooth kernel, Proc. Amer. Math. Soc. 103 (1988), no. 1, 260-264.

[11] T.P. Hytönen, L. Roncal and O. Tapiola, Quantitative weighted estimates for rough homogeneous singular integrals, Israel J. Math., 218 (2017), no. 1, 133164.

[12] M.T. Lacey, An elementary proof of the $A_{2}$ bound, Israel J. Math., 217 (2017), no. 1, 181-195.

[13] M.T. Lacey, Sparse bounds for spherical maximal functions, preprint. Available at https://arxiv.org/abs/1702.08594, to appear in J. Anal. Math.

[14] M.T. Lacey and S. Spencer, Sparse bounds for oscillatory and random singular integrals, New York J. Math., 23 (2017), 119-131.

[15] A.K. Lerner, On pointwise estimates involving sparse operators, New York J. Math., 22 (2016), 341-349.

[16] A.K. Lerner and F. Nazarov, Intuitive dyadic calculus: the basics, preprint. Available at http://arxiv.org/abs/1508.05639

[17] A. Seeger, Singular integral operators with rough convolution kernels, J. Amer. Math. Soc. 9 (1996), no. 1, 95-105.

Department of Mathematics, Bar-Ilan University, 5290002 Ramat GAN, ISRAEL

E-mail address: lernera@math.biu.ac.il 